\title{
Higher Performance of QuantiFERON TB Compared to Tuberculin Skin Test in Latent Tuberculosis Infection Prospective Diagnosis
}

\author{
Tarak Dhaouadi ${ }^{1}$, Imen Sfar ${ }^{1}$, Leila Mouelhi ${ }^{2}$, Raoudha Tekaya ${ }^{3}$, Ines Mahmoud ${ }^{3}$, \\ Jalila Bargaoui ${ }^{1}$, Raoudha Daghbouj ${ }^{1}, \quad$ Leila Abdelmoula ${ }^{3}$, Taoufik Najjar ${ }^{2}$, \\ Taieb Ben Abdallah ${ }^{1}$ and Yousr Gorgi, ${ }^{*}$
}

${ }^{1}$ Laboratory of Research in Immunology of Renal Transplantation and Immunopathology (LR03SP01), Charles Nicolle Hospital, Tunis El Manar University, Tunis, Tunisia

${ }^{2}$ Gastroenterology Department, Charles Nicolle Hospital, Tunis El Manar University, Tunis, Tunisia

${ }^{3}$ Rheumatology Department, Charles Nicolle Hospital, Tunis El Manar University, Tunis, Tunisia

\begin{abstract}
Background: The Tuberculin skin test (TST) has been used for years in the latent tuberculosis infection (LTBI) diagnosis, but it has, well-documented, low sensitivity and specificity. Interferon-y release assays (IGRA) has been reported to be more sensitive and specific than TST. Therefore, this study aimed to evaluate the performance of a commercial IGRA, QuantiFERON ${ }^{\circledR}$-TB Gold In-Tube (QFT-GIT), comparatively to TST in LTBI diagnosis.

Patients and Methods: This study included 238 patients who were candidate for an anti-TNF therapy. The screening for LTBI was performed by both TST and QFT-GIT test for all patients. In order to evaluate the strength of associations, the odds ratios $(\mathrm{OR})$ together with $95 \%$ confidence intervals $(\mathrm{Cl})$ were calculated. The correlation between QFT-GIT and TST was evaluated using $\mathrm{k}$ statistics.

Results: Sixty-three (26.4\%) sera were positive for QFT-GIT with a mean level of IFN-ץ of about $1.18 \mathrm{IU} / \mathrm{ml}$, while 81 (34\%) patients were positive for TST. Agreement between QFT-GIT and TST was poor (37 QFT-GIT+/TST- and 55 QFTGIT-/TST+), $\quad K=0.09$ (SD=0.065). The positivity of QFT-GIT was not influenced by $B C G$ vaccination or by immunosuppression. Nevertheless, it was significantly associated to both history of an earlier tuberculosis disease (HETD) and its radiological sequel (RS), $p=6 \mathrm{E}-7$ and $p=1 \mathrm{E}-8$, respectively. Inversely, the TST results were not correlated to either HETD or RS, but the TST positivity was less frequent in immunosuppressed patients $(45.5 \%$ vs. $73.9 \%), p=1 \mathrm{E}$ 5 , OR $(95 \% \mathrm{Cl})=0.29[0.17-0.52]$. Moreover, the extent of both the immunosuppression period and the time elapsed from the last BCG injection was significantly correlated to a lesser TST positivity, $p=3 \mathrm{E}-12$ and $p=5 \mathrm{E}-7$, respectively. Among the QFT-GIT-/TST+ patients $(n=55)$ whom received an anti-TNF agent without any prophylactic treatment of LTBI, no tuberculosis was detected with a median follow-up of 78 weeks [56-109].
\end{abstract}

Conclusion: Our study suggests that the QFT-GIT has a higher performance comparatively to TST in the LTBI screening that is unaffected by either BCG vaccination or immunosuppression. Therefore, IGRAs has to replace TST especially in patients who are under consideration for an anti-TNF therapy.

Keywords: Tumor necrosis factor- $\alpha$ inhibitors, latent tuberculosis infection, Tuberculin skin test, QuantiFERON-TB Gold In-Tube.

\section{INTRODUCTION}

The tumor necrosis factor- $\alpha$ (TNF- $\alpha$ ) is a proinflammatory, multifunctional cytokine secreted by a variety of cells including activated macrophages and Tcells [1]. The TNF- $\alpha$ effect consists of activation of neutrophils, macrophages and natural killer (NK) cells, increasing cell adhesion molecules expression and inducing cytotoxicity against failing infected macrophages [1]. Although there is no invading microbe, elevation of TNF- $\alpha$ amount have been detected during several chronic inflammatory conditions such as autoimmune rheumatic diseases $(A R D)$ and inflammatory bowel diseases (IBD). Hence,

${ }^{*}$ Address correspondence to this author at the Laboratory of Research in Immunology of Renal Transplantation and Immunopathology (LR03SP01), University Tunis El Manar, Charles Nicolle Hospital, Bd 9 Avril, 1006 Bab Saadoun, Tunis, Tunisia; Tel: +21698341791; Fax: +216 71561156;

E-mail: gorgi.yousr@gmail.com it has been proposed that blockade of TNF- $\alpha$ is able to improve these chronic autoimmune diseases. In fact, the anti-TNF- $\alpha$ therapies had so revolutionized their courses that led to increase their clinical use and resulted inevitably in a related increase of adverse effects [1]. Among these adverse effects, there is an increased incidence of opportunistic infections such as reactivation of latent tuberculosis infection (LTBI). The tuberculosis (TB) infection results in granulomas composed of epithelioid macrophages and multinucleated giant cells that are bordered by T-cells and associated to a central necrosis. The initial cytokine response to Mycobacterium Tuberculosis (MT), including TNF- $\alpha$, IL-12 and chemokines, is triggered by direct interaction of mycobacterial antigens with toll-like receptors of macrophages [2]. Mostly, this initial innate immune response fails to control the MT replication. Therefore, adaptive immune cells, CD4 ${ }^{+}$ and $\mathrm{CD}^{+} \mathrm{T}$-cells, migrate to the site of infection trying 
to contain the MT by promoting the formation of mature granuloma [2]. TNF- $\alpha$ plays a crucial role in this process as its neutralization decreases both recruitment of inflammatory cells and granuloma formation [2]. Moreover, TNF- $\alpha$ is required for instituting and preserving granuloma architecture: it regulates the tight association between macrophages and T-cells within granulomas [2].

One-third of the world's population, more than 2 billion people, is believed to harbor LTBI [3]. The TB is endemic in Tunisia and its incidence is currently estimated at 32/100,000 [3]. In 1956, the anti-TB efforts began in Tunisia by TB-screening campaigns [4]. Later in 1978, the anti-TB program was born with widespread $B C G$ vaccination and gratis treatment of TB until complete recovery [4]. Nevertheless, the global efficiency of this program varied between $26 \%$ and $31 \%$ due in part to a low effectiveness of screening methods estimated at only $61 \%$ [4]. Based on this observation, the LTBI prevalence in Tunisia may even surpass the world's average estimated at one-third. Therefore, screening for LTBI has become mandatory prior to the initiation of the TNF- $\alpha$ inhibitor therapy. However, diagnosis of LTBI is problematic, particularly in immunosuppressed patients since neither the targeted imaging techniques nor the tuberculin skin test (TST) are sufficiently accurate. TST attempts to measure cell-mediated immunity in the form of delayed-type hypersensitivity response to purified protein derivative (PPD). Nevertheless, TST lacks specificity for MT as either former exposure to environmental mycobacteria or prior BCG vaccination are both providers of false-positive results.

The identification of the specific immunogenic MT proteins ESAT-6, CEP-10 and TB-7.7 has led to the development of more specific diagnostic assays for TB disease and LTBI. IFN- $y$ release assays (IGRAs) try to exploit the response of previously primed MT-specific T-cells. Among IGRAs, the QuantiFERON ${ }^{\circledR}$-TB Gold InTube (QFT-GIT) is a whole blood test, which offer many advantages in comparison to TST. In fact, QFTGIT is theoretically not influenced by the BCG vaccination, exempts from a $2^{\text {nd }}$ visit to read TST and therefore it is cost-effective compared to the TST [5]. Furthermore, IGRAs are more sensitive and specific than TST in the diagnosis of active bacillary TB [6]. However, these performances cannot be extrapolated to LTBI. Indeed, the bacillary load is different between $T B$ and $L T B I$, hence we ignore if T-cell reactivity is quantitatively, qualitatively and kinetically identical or not. Moreover, a QFT-GIT positive result cannot differentiate between TB disease and LTBI. In this case, the diagnosis of $L T B I$ requires that TB disease is being excluded by medical evaluation, chest X-Ray and examination of sputum. Eventually, as long as there is no "gold standard" for LTBI diagnosis evaluation of IGRAs performances will remain problematic. In this context, we performed a longitudinal study in order to estimate predictive values of QFT-GIT in a Tunisian cohort of patients who were candidates to an anti-TNF therapy.

\section{MATERIAL AND METHODS}

\section{Patients and Controls}

This study included 238 patients followed-up for chronic inflammatory conditions. Patients were visiting both rheumatology and gastroenterology departments of the Charles Nicolle Hospital in Tunis. All patients were candidate for anti-TNF therapy for either ARD or IBD. Active bacillary TB diagnosis was eliminated before the start of the anti-TNF therapy by clinical check, chest X-Ray and analysis of sputum (direct smear examination and culture in Lowenstein-Jensen medium). Among the initial group, which involved 241 patients, $3(1.24 \%)$, had indeterminate result of QFTGIT and were definitely excluded from this study. Clinical, biological and radiological features of enrolled patients are recorded in Table 1.

All patients gave written informed consent to participate in the study, and patient anonymity was preserved using documents and methods approved by the local Ethics committee of Charles Nicolle Hospital.

\section{Methods}

\section{QuantiFeron $^{\circledR}$-TB Gold In-Tube (QFT-GIT) Assay}

The QFT-GIT test was processed according to the manufacturer's recommendations. The manufacturer recommends to use four standards: $4 \mathrm{IU} / \mathrm{ml}, 1 \mathrm{IU} / \mathrm{ml}$, $0.25 \mathrm{IU} / \mathrm{ml}$ and $0 \mathrm{IU} / \mathrm{ml}$. In order to quantify IFN-y levels that are higher than $4 \mathrm{IU} / \mathrm{ml}$ we performed a five standard assay by adding an $8 \mathrm{lU} / \mathrm{ml}$ standard $(50 \mu \mathrm{l}$ of the kit standard without the green diluent). The QFTGIT is considered positive when the IFN- $y$ level is $\geq$ $0.35 \mathrm{IU} / \mathrm{ml}$ (after deducting the nil well value) and $\geq$ of about $25 \%$ than the negative control well value. The result is considered negative if the IFN-y level is $<0.5$ $\mathrm{IU} / \mathrm{ml}$ and the positive control value is $\geq 0.5 \mathrm{IU}$ (after deducting the nil well value). Indeterminate results with IFN-y rate $<0.35 \mathrm{IU} / \mathrm{ml}$ and positive control $<0.5 \mathrm{IU} / \mathrm{ml}$ were excluded from this study. 
Table1: Clinical, Biological and Radiological Features of Enrolled Patients

\begin{tabular}{|c|c|}
\hline Patients & N=238 \\
\hline \hline Age \pm SD (years) & $47.4 \pm 14.58$ \\
\hline Sex Ratio (men/women) & $0.525(82 / 156)$ \\
\hline History of an earlier TB disease n (\%) & 25 (10.5\%) \\
\hline BCG-vaccinated & $33.98 \pm 19.69$ \\
\hline Period between last BCG injection and LTBI screening (years) & $160(67.2 \%)$ \\
\hline ARD n (\%) & 78 (32.7\%) \\
\hline IBD n (\%) & $153(64.3 \%)$ \\
\hline Immunosuppression* & 48 [12-182.25] \\
\hline Median immunosuppression duration [1st - 3rd Quartiles] (months) & 78 [56-128] \\
\hline Median follow-up [1 ${ }^{\text {st }}$ - ${ }^{\text {rd }}$ Quartiles] (weeks) & 0 \\
\hline Sputum examination positive & 81 (34\%) \\
\hline TST positive & $18(7.6 \%)$ \\
\hline Sequel images of ancient TB disease in chest X-Ray & \\
\hline
\end{tabular}

*ARD: steroids + methotrexate; IBD: methotrexate.

\section{Tuberculin Skin Test (TST)}

TST was performed in all patients by trained technicians on the volar side of the forearm by intradermal injection of $2 T U / 0.1 \mathrm{ml}$ of PPD. The interpretation of TST result was made 72 hours after the PPD injection. TST was interpreted according to the Tunisian recommendations, in which the threshold is $5 \mathrm{~mm}$ when the person is at high risk for TB disease or immunosuppressed and $10 \mathrm{~mm}$ in case of low TB risk [7].

In order to avoid the risk of cross reactivity TST was executed after the sampling for QFT-GIT test, and was interpreted blindly by skilled physicians.

\section{Statistical Analysis}

The results of continuous quantitative variables (Age, period between the last BCG injection and QFTGIT assay, immunosuppression duration and follow-up period) are expressed as means $\pm S D$, and the means of groups were compared by ANOVA-test (SPSS 11 Inc. Chicago, Illinois, USA). For qualitative variables, univariable analysis was performed using chi-square test or fisher's exact test for small numbers (SPSS 11 Inc. Chicago, Illinois, USA). Values of $p<0.05$ were considered to be statistically significant.

In order to evaluate the strength of associations, the odds ratios (OR) together with 95\% confidence intervals $(\mathrm{Cl})$ were calculated. Logistic regression models were built according to gender, BCG vaccination status and immunosuppression to estimate adjusted ORs. The built models included all these factors in the same time by stratification. Without a "gold standard" for LTBI diagnosis, it is impossible to determine the sensitivity and specificity of both QFTGIT and TST. Instead, we compared the performance of QFT-GIT and TST in relation to the BCG vaccination status and the period elapsed from the last injection, history of a prior TB disease, immunosuppression and its duration and the presence of radiological sequel of a previous TB disease. The correlation between QFTGIT and TST was evaluated using $\mathrm{k}$ statistics (SPSS 11 Inc. Chicago, Illinois, USA). Based on the $\mathrm{k}$ test, the agreement is poor when it ranges between 0 and 0.2 , fair between 0.2 and 0.4 , moderate between 0.4 and 0.6 , substantial between 0.6 and 0.8 , and almost perfect between 0.8 and 1 . When $\mathrm{K}$ is $<0$ the tests results are considered contradictory.

\section{RESULTS}

In total, 238 patients were included for whom data from the sputum examination, the chest X-Ray and TST were available (Table 1). Mean age of the studied group was at $47.4 \pm 14.58$ years with a sex ratio (men/women) of 0.525 (82/156). Two hundred and twenty-eight $(95.8 \%)$ subjects underwent BCG vaccination during childhood. Among them, 67.2\% suffered from ARD and $32.7 \%$ from IBD. At the time of LTBI screening 153 (64.3\%) patients were under immunosuppressive therapy at a median duration of 48 weeks [12-182.25]. Twenty-five (10.5\%) patients had a 
Table 2: Analytic Results of QFT-GIT Test

\begin{tabular}{|c|c|c|c|c|}
\hline & $\mathrm{QFT}+(\mathrm{n}=63)$ & QFT - (n=175) & $p$ & OR (95\%Cl) \\
\hline History of previous TB disease & $17(27 \%)$ & $8(4.6 \%)$ & $6 \mathrm{E}-7$ & $7.71^{*}[3.13-19]$ \\
\hline BCG-vaccinated & $59(93.7 \%)$ & $169(96.6 \%)$ & 0.322 & - \\
\hline Period between last BCG injection and QFT-GIT (years) & 34.93 & 33.65 & 0.671 & - \\
\hline Immunosuppression & $39(61.9 \%)$ & $114(65.1 \%)$ & 0.646 & - \\
\hline Immunosuppression duration (months) & 98.23 & 95.98 & 0.902 & - \\
\hline Sequel images of ancient TB disease in chest X-Ray & $15(23.8 \%)$ & $3(1.7 \%)$ & $1 \mathrm{E}-8$ & $17.9^{\dagger}[4.9-64.4]$ \\
\hline
\end{tabular}

${ }^{*}$ ORs: 1) a/ Layer immunosuppression (-)/BCG-Vaccinated/Males = 2.7; b/ Layer immunosuppression $(-) /$ BCG-Vaccinated/Females = 21; c/ Total (Males \& Females $)=7.071 ; 2)$ Layer Immunosuppression $(+) /$ BCG-Vaccinated/Males $=1.5 ;$ Layer immunosuppression $(+) /$ BCG-Vaccinated/Females $=6.87 ;$ Total $($ Males $\&$ Females) $=12.91$.

†ORs: 1) a/ Layer immunosuppression (-)/BCG-Vaccinated/Males = 8.7; b/ Layer immunosuppression (-)/ BCG-Vaccinated/Females = 19.33; c/ Total (Males \& Females $)=12.68 ; 2)$ Layer Immunosuppression $(+) /$ BCG-Vaccinated/Males = 1.5; Layer immunosuppression $(+) /$ BCG-Vaccinated/Females $=13.91 ;$ Total $($ Males \& Females) $=26.09$.

history of a previous treated TB disease while its radiological sequel (nodular densities, fibrotic scars, calcified granulomas or basal pleural thickening) were found in only $18(7.6 \%)$ subjects. None of the 238 enrolled patients had any clinical, biological or radiological evidence of an active TB disease.

\section{QFT-GIT Results}

The QFT-GIT test was positive in 63 (26.4\%) patients with a mean level of IFN- $\gamma=1.11 \mathrm{IU} / \mathrm{ml}$ (mean level for positive $=4.44 \mathrm{IU} / \mathrm{ml}$ and for negative $=0.0051$ $\mathrm{IU} / \mathrm{ml})$. QFT-GIT was positive in $68 \%$ of patients with old healed TB and in $83.3 \%$ of those with radiographic lesions. Consequently, QFT-GIT positivity was significantly associated with history of a previous TB disease and its radiological sequel; $p=6 \mathrm{E}-7$ OR $(95 \%$ $\mathrm{Cl})=7.71[3.13-19]$ and $p=1 \mathrm{E}-9$ OR $(95 \% \mathrm{Cl})=17.9$ [4.9-64.4], respectively (Table 2 ).

In addition, IFN-y levels were significantly higher in case of ancient TB disease (5.6 vs. $0.66 \mathrm{IU} / \mathrm{ml}$ ) and in presence of radiological sequel (5.66 vs. $0.81 \mathrm{IU} / \mathrm{ml}$ ), $p=2 \mathrm{E}-12$ and $p=5 \mathrm{E}-9$, respectively (Table 3 ).

Inversely, QFT-GIT positivity and IFN-y levels were not correlated to either BCG vaccination status, or the immunosuppression and its duration (Tables 2 and $\mathbf{3}$ ).

\section{TST Results}

Eighty-one (34\%) patients had a positive TST. TST positivity was significantly correlated to BCG vaccination, $p=0.02$ OR $(95 \% \mathrm{Cl})=1.55[1.4-1.7]$ (Table 4). Mean of period between last BCG injection and LTBI screening was significantly lower in case of positive TST (22.44 vs. 40.52 years), $p=3 \mathrm{E}-12$. The studied cohort was then subdivided into 2 groups: 1) G1: period between last BCG vaccination and LTBI screening $<30$ years $(n=108)$ and 2) G2: period $\geq 30$ years $(n=130)$. TST was significantly more frequently positive in G1 (80.2\%) comparatively to G2 (27.3\%), $p=5 \mathrm{E}-13$ OR $(95 \% \mathrm{Cl})=9.4$ [4.9-18.1]. Besides, the TST positivity was significantly less frequent in

Table 3: Analytic Result of IFN- $y$ Levels

\begin{tabular}{|c|c|c|c|}
\hline & & Mean IFN-Y level & $p$ \\
\hline \multirow{2}{*}{ History of previous TB disease } & Present & 5.6 & \multirow{2}{*}{$2 \mathrm{E}-12$} \\
\hline & Absent & 0.66 & \\
\hline \multirow{2}{*}{ BCG vaccination } & Present & 0.88 & \multirow{2}{*}{0.782} \\
\hline & Absent & 1.19 & \\
\hline \multirow{2}{*}{ Immunosuppression } & Present & 0.98 & \multirow{2}{*}{0,26} \\
\hline & Absent & 1.52 & \\
\hline \multirow{2}{*}{ TST } & Positive & 1.38 & \multirow{2}{*}{0.51} \\
\hline & Negative & 1.07 & \\
\hline \multirow{2}{*}{ Sequel images of ancient TB disease in chest X-Ray } & Present & 5.66 & \multirow{2}{*}{$5 \mathrm{E}-9$} \\
\hline & Absent & 0.81 & \\
\hline
\end{tabular}


Table 4: Analytic Results of TST

\begin{tabular}{|c|c|c|c|c|}
\hline & $\mathrm{TST}+(\mathrm{n}=81)$ & TST $-(n=157)$ & $p$ & OR (95\%Cl) \\
\hline History of previous TB disease & $11(13.6 \%)$ & $14(8.9 \%)$ & 0.266 & - \\
\hline BCG-vaccinated & $81(100 \%)$ & $147(93.6 \%)$ & 0.02 & $1.55[1.4-1.7]$ \\
\hline Period between last BCG injection and TST (years) & 22.44 & 40.52 & $3 \mathrm{E}-12$ & - \\
\hline Period between last BCG injection and TST $<30$ years & $65(80.2 \%)$ & $43(27.3 \%)$ & $5 E-13$ & $9.4[4.9-18.1]$ \\
\hline Immunosuppression & $37(45.7 \%)$ & $116(73.9 \%)$ & $1 \mathrm{E}-5$ & $0.29[0.17-0.52]$ \\
\hline Immunosuppression duration (months) & 30.24 & 118.28 & $5 \mathrm{E}-7$ & - \\
\hline Sequel images of ancient TB disease in chest X-Ray & $9(11.1 \%)$ & $9(5.7 \%)$ & 0.137 & - \\
\hline
\end{tabular}

immunosuppressed patients (47.5\% vs. $73.9 \%), p=1 \mathrm{E}-$ 5 OR $(95 \% \mathrm{Cl})=0.29[0.17-0.52]$ (Table 4). Moreover, the mean immunosuppression duration was significantly lower in positive TST patients (30.24 vs. 118.28 months), $p=5 \mathrm{E}-7$ (Table 4).

Inversely, the TST positivity was not correlated to either history of ancient TB disease or the presence of radiological sequel (Table 4 ). In fact, TST was positive in only $44 \%$ of patients with history of treated TB and $50 \%$ of those with radiological lesions.

\section{Agreement between QFT-GIT and TST}

The overall agreement between QFT-GIT and TST was poor, $\mathrm{K}=0.09(\mathrm{SD}=0.065)$, with concordant results in only $146(61.3 \%)$ patients (Table 5). The discordant results were 37 (15.5\%) QFT-GIT+/TSTand 55 (23.1\%) QFT-GIT-/TST+. Moreover, the TST results were not correlated to the level of IFN-Y (Table 3). Analysis of the agreement between QFT-GIT and TST revealed no influence of BCG vaccination status, immunosuppression, history of prior TB disease and its radiological squeal as all $\mathrm{K}$ values remained poor
(Table 5). Contrariwise, the agreement was better in $\mathrm{G} 2(\mathrm{~K}=0.311)$ than in $\mathrm{G} 1(\mathrm{~K}=0.018)$.

\section{Analysis of Discrepancies between QFT-GIT and TST}

The whole QFT-GIT/TST disagreement was not correlated to either BCG vaccination status, immunosuppression, history of previous TB disease or its radiological sequel; $p=0.929, p=0.153, p=0.6$ and $p=0.31$, respectively. The QFT-GIT+/TST- discordance was significantly correlated to ancient healed TB $(36 \%$ vs. $13.1 \%)$ and radiological lesions (38.9\% vs. $13.6 \%)$, $p=0.003$ OR $(95 \% \mathrm{Cl})=3.71[1.49-9.21]$ and $p=0.004$ OR $(95 \% \mathrm{Cl})=4.03$ [1.44-11.2], respectively. Inversely, the QFT-GIT-/TST+ discordance was negativelyassociated with immunosuppression (17\% vs. $34.1 \%$ ), $p=0.003$ OR $(95 \% \mathrm{Cl})=0.395[0.214-0.732]$.

\section{Follow-Up}

Patients with QFT-GIT positive result ( $n=63,26.4 \%$ ) received a prophylactic treatment of LTBI for 3 months: Rifampicin $10 \mathrm{mg} / \mathrm{kg}$ in association with Isoniazid 5

Table 5: Agreement between QFT-GIT and TST

\begin{tabular}{|c|c|c|c|c|}
\hline \multicolumn{2}{|l|}{ Parameter } & QFT-GIT+/TST- & QFT-GIT-/TST+ & $\mathrm{K}(\mathrm{SD})$ \\
\hline \multirow{2}{*}{ BCG-vaccinated } & Yes & 33 & 55 & $0.103(0.066)$ \\
\hline & No & 4 & 0 & 0 \\
\hline \multirow{2}{*}{$\begin{array}{c}\text { Period between last BCG injection and LTBI screening }<30 \\
\text { years }\end{array}$} & Yes & 9 & 50 & $0.018(0.07)$ \\
\hline & No & 23 & 5 & $0.311(0.094)$ \\
\hline \multirow{2}{*}{ Immunosuppression } & Yes & 28 & 26 & $0.055(0.083)$ \\
\hline & No & 9 & 29 & $0.119(0.095)$ \\
\hline \multirow{2}{*}{ History of previous TB disease } & Yes & 9 & 3 & $0.08(0.176)$ \\
\hline & No & 28 & 52 & $0.067(0.068)$ \\
\hline \multirow{2}{*}{ Sequel images of ancient TB disease in chest X-Ray } & Yes & 7 & 1 & $0.111(0.175)$ \\
\hline & No & 30 & 54 & $0.052(0.066)$ \\
\hline \multicolumn{2}{|l|}{ Overall } & 37 (15.5\%) & 55 (23.1\%) & $0.09(0.065)$ \\
\hline
\end{tabular}


$\mathrm{mg} / \mathrm{kg}$ daily. Three weeks after the beginning of the prophylaxis, TNF-inhibitory treatment started. After the initiation of anti-TNF therapy, patients underwent a periodical follow-up at 3 months, 6 months, 12 months, 18 months, 2 years and 3 years. The periodical followup comprised clinical, biological and radiological examinations.

Aiming to determine the negative predictive value (NPV) of QFT-GIT for LTBI, all patients with negative IGRA $(n=175,73.4 \%)$ even in the case of a positive TST $(n=55,23.1 \%)$ had not received any prophylactic treatment of LTBI and had been followed-up periodically as mentioned before. No tuberculosis disease occurred with a median follow-up of 78 weeks [56-109], thus the NPV of QFT-GIT for LTBI occurrence was at $100 \%$.

\section{DISCUSSION}

In the present study, we prospectively enrolled 238 patients monitored and treated or newly diagnosed for chronic inflammatory conditions in whom active TB disease was excluded. All patients underwent QFT-GIT test then TST and were followed-up for 78 months [56109] months to detect LTBI reactivation.

In the present study, we confirmed the higher performance of QFT-GIT in comparison to TST in LTBI screening:

1) The QFT-GIT test was positive in 63 (24.6\%) patients, which is in agreement with the estimated prevalence of LTBI by WHO [3]. This prevalence corroborates those found in other studies performed in populations with medium-low incidence of TB disease [8-9]. Inversely, in populations with low TB incidence, the positivity of IGRAs was considerably lower [10-12]. Besides, the positivity of QFT-GIT and IFN-y levels were not influenced by either BCG vaccination or immunosuppression and its duration. In this regard, literature data are controversial. In fact, if some studies revealed that immunosuppressive therapy had no impact on IGRAs results [8, 12-14], others showed that it might moderately decrease their positivity $[11,15]$. In contrast, most of published studies revealed that the $B C G$ vaccination did not influence the positivity of QFTGIT $[12,13,16]$.

The analytic results in our study indicated that QFTGIT positivity and IFN-y levels were correlated to both history of a previous well-treated TB disease and its radiological sequel. This is in agreement with several findings in literature, which confirmed that QFT-GIT remains positive after active TB disease treatment or LTBI prophylactic therapy [17-21]. This issue might be due to the persistence of the TB-specific memory Tcells that may only mean a biological scar of a prior contact with MT. Consequently, the absence of IFN-Y reverse after the treatment of LTBI and TB disease may affect the specificity of this test in the LTBI screening. However, it has been reported that radiographic lesions consistent with old healed TB is one of the strongest factors for development of active TB [22]. In another report, these radiological scars of treated TB were associated with 8.8-folder higher OR for the subsequent development of active TB [23]. Therefore, and by corollary, our results suggest a good sensitivity of QFT-GIT in LTBI screening, albeit impossible to be accurately calculated.

2) TST was positive in 81 (34\%) patients. The analysis of risk factors for TST positivity revealed a positive correlation with BCG vaccination and a shorter period between the last vaccine injection and the LTBI screening. This observation corroborates the results of a meta-analysis, which concluded that BCG vaccination, and the non-tuberculous mycobacteria were responsible for false-positive TST [24]. Moreover, Babayigit et al. [9] observed that positivity of TST and the diameter of the induration were significantly correlated to the number of BCG scar. Consequently, BCG vaccination especially if there was multiple booster injections within the last 30 years is a source of false-positive TST. Besides, TST was negatively correlated to immunosuppression and its duration. The adverse impact of immunosuppression on BCG positivity was also observed in several other published studies $[8,12,14,25]$. Thus, the sensitivity of TST in detecting LTBI is directly affected by immunosuppressive therapy. Against all odds, we found no correlation between history of ancient treated TB or radiological TB-sequel and the positivity of TST. This was equally the case in the study of Matulis et al. [11]. This issue can be explained by the presence of IgG class tuberculin specific antibodies that can neutralize it. In fact, IgG Tuberculin antibodies were found to be associated with blocking of in vitro cultures of PBMC with Tuberculin [26]. Therefore, the presence of such protective [26] antibodies would be predictive of false negative TST.

3) In our study and as expected, the results of QFTGIT and TST were globally discordant, $\mathrm{K}=0.09$. Moreover, the agreement remained poor even after stratification with BCG vaccination, 
immunosuppression, history of a previous treated TB disease and chest X-Ray sequel. In contrast, extent of the period from the last BCG injection improved moderately the agreement between the 2 tests, $\mathrm{K}=0.311$. The poor concordance between QFT-GIT and TST was largely observed in literature especially in BCG-vaccinated patients [8, 11, 13-16]. Inversely, the agreement between QFT-GIT was moderate in populations with a low rate of BCG vaccination [12], and substantial in non-vaccinated patients $[13,16]$. Eventually, the discrepancies between QFT-GIT and TST are mostly due to the impact of either BCG or the immunosuppressive therapy on TST while these factors did not seem to influence the IGRA results.

4) To estimate negative predictive value (NPV) of QFT-GIT, the 175 patients with negative IGRA even in case of a positive TST $(n=55)$ did not receive the prophylactic treatment of LTBI. No tuberculosis occurred under the anti-TNF therapy within a median follow up of 78 weeks. Considering that the most cases of TB in patients receiving anti-TNF drugs are detected within 12 months (52 weeks) [1, 2], our findings gave a clue about the excellent NPV of QFT-GIT. Likewise, Laffite et al. [27] and Pratt et al. [28] showed that even in immunosuppressed patients with negative IGRA, no TB reactivation happened within 76 weeks and 18.3 months of follow-up, respectively.

In spite of the insufficiency of these data, many countries formulated recommendations that were more or less relieved by official bodies, proposing three types of approach, sometimes multiple according to the risk groups, sometimes specific to the consideration for anti-TNF therapy [13]:

a) The IGRAs has to replace the TST: Germany, Switzerland, Poland, and Denmark. Only under consideration for anti-TNF- $\alpha$ therapy: Italy, Netherland and Japan.

b) The TST or the IGRAs can be indifferently used: USA, Australia, France, Japan, and Denmark.

c) The TST must be used by first intention, completed in case of doubtful result by an IGRA to try to improve its sensibility and its specificity: Canada, United Kingdom, Italy, Spain, Norway, Czech Republic, and Korea.

The recent consensus recommendations provided by the European Tuberculosis Network Trials Group (TBNET) are: both TST and IGRA test should be done to all patients except BCG-vaccinated patients for whom a TST is unnecessary; the treatment for LTBI is recommended if any of the tests are positive [13].

Considering the fact that the BCG vaccination is mandatory in Tunisia, the TST should be used only when the period from the last BCG booster injection exceeds 30 years and if the patient is not immunosuppressed. If the TST is negative, an IGRA should be performed to eliminate the false negative TST results. Otherwise, an IGRA test is recommended. If the patient is under consideration for an anti-TNF therapy, the IGRAs has to replace the TST.

\section{CONCLUSION}

Our study suggests that the QFT-GIT has higher performance comparatively to the TST in the LTBI screening that is unaffected by either the BCG vaccination or the immunosuppression. The TST can be used only if the last BCG injection is far in time (> 30 years). Otherwise, the IGRAs has to replace the TST especially in patients who are candidates for antiTNF therapy.

\section{ACKNOWLEDGEMENTS}

This work was granted and supported by the Laboratory of Research in Immunology of Renal Transplantation and Immunopathology (LR03SP01). University Tunis El Manar. Charles Nicolle Hospital. Tunis, TUNISIA

\section{COMPETING INTERESTS}

No benefits in any form have been received or will be received from a commercial party related directly or indirectly to the subject of this article.

\section{AUTHORS' CONTRIBUTION}

Pr Gorgi Yousr proposed the study. Tarak Dhaouadi and Imen Sfar analyzed the data. All authors contributed to the design and interpretation of the study. Pr Gorgi Yousr is the guarantor of the integrity of this study.

\section{REFERENCES}

[1] Connor V. Anti-TNF therapies: a comprehensive analysis of adverse effects associated with immunosuppression. Rheumatol Int 2011; 31: 327-37. http://dx.doi.org/10.1007/s00296-009-1292-x

[2] Furst DE, Wallis R, Broder M, Beenhouwer DO. Tumor necrosis factor antagonists: Different kinetics and/or mechanisms of action may explain differences in the risk for 
developing granulomatous infection. Semin Arthritis Rheum 2006; 36: 159-67.

http://dx.doi.org/10.1016/j.semarthrit.2006.02.001

[3] World Health Organization. Global Tuberculosis report 2014. WHO/HTM/TB/2014.08 http://www.who.int/tb/publications/ global_report/en/

[4] Chahed MK, Bellali H, Dhouibi S, Ben Alaya N, Zouari B. Tuberculosis control programme in Tunisia: efficacy assessment. Sante 2010; 20: 87-92.

[5] Iqbal AZ, Leighton J, Anthony J, Knaup RC, Peters EB, Bailey TC. Cost-effectiveness of using Quantiferon gold $\left(\right.$ QFT-G) ${ }^{\circledR}$ versus tuberculin skin test (TST) among U.S. and foreign born populations at a public health department clinic with a low prevalence of tuberculosis. Public Health Nurs 2014; 31: 144-52. http://dx.doi.org/10.1111/phn.12083

Diel R, Loddenkemper R, Nienhaus A. Evidence-based comparison of commercial interferon-gamma release assays for detecting active TB: a metaanalysis. Chest 2010; 137: 952-68.

http://dx.doi.org/10.1378/chest.09-2350

Siturk L. Haut Conseil de la santé publique. Les nouveaux tests de dépistage de la tuberculose. http://213.150.177.30/mn/details_article.php?t=530\&a=23335 \&temp=1\&lang=

[8] Kim JH, Cho SK, Han M, Choi CB, Kim TH, Jun JB, et al. Factors influencing discrepancies between the QuantiFERON-TB Gold in Tube test and the tuberculin skin test in Korean patients with rheumatic diseases. Semin Arthritis 2013; 42: 424-32.

http://dx.doi.org/10.1016/j.semarthrit.2012.07.001

[9] Babayigit C, Ozer B, Tacettin O, Ozer C, Duran N, Gocmen O. Performance of QuantiFERON-TB Gold In-Tube test and tuberculin skin test for diagnosis of latent tuberculosis infection in BCG vaccinated health care workers. Med Sci Monit 2014; 20: 521-9. http://dx.doi.org/10.12659/MSM.889943

[10] Ramos JM, Robeldano C, Masiá M, Belda S, Padila S, Rodriguez JC, et al. Contribution of interferon gamma release assays testing to the diagnosis of latent tuberculosis infection in HIV-infected patients: A comparison of QuantiFERON-TB gold in tube, T-SPOT.TB and tuberculin skin test. BMC Infect Dis 2012; 12: 169. http://dx.doi.org/10.1186/1471-2334-12-169

[11] Matulis G, Jüni P, Villiger PM, Gadola SD. Detection of latent tuberculosis in immunosuppressed patients with autoimmune diseases: performance of a Mycobacterium tuberculosis antigen-specific interferon y assay. Ann Rheum Dis 2008; 67: 84-90. http://dx.doi.org/10.1136/ard.2007.070789

[12] Bartalesi F, Vicidomini S, Goletti D, Fiorelli C, Fiori G, Melchiorre D, et al. QuantiFERON-TB gold and TST are both useful for latent tuberculosis infection screening in autoimmune diseases. Eur Respir J 2009; 33: 586-93. http://dx.doi.org/10.1183/09031936.00107608

[13] García-Gasalla M, Fernández-Baca V, Juan-Mas A, PayerasCifre A, Cifuentes-Luna C, Tberner-Ferrer R, et al. Use of Quantiferon-TB-Gold in Tube® test for detecting latent tuberculosis in patients considered as candidates for antiTNF therapy in routine clinical practice. Enferm Infecc Microbiol Clin 2013; 31: 76-81.

http://dx.doi.org/10.1016/i.eimc.2012.06.014

[14] Duman N, Ersoy-Evans S, Karadağ Ö, Aşçioğlu S, Şener B, Kiraz $\mathrm{S}$, et al. Screening for latent tuberculosis infection in psoriasis and psoriatic arthritis patients in a tuberculosisendemic country: a comparison of the QuantiFERON ${ }^{\circledR}$-TB Gold In-Tube test and tuberculin skin test. Int J Dermatol 2014; 53: 1286-92.

http://dx.doi.org/10.1111/ijd.12522
[15] Papay P, Eser A, Winkler S, Frantal S, Primas C, Miehsler $W$, et al. Factors impacting the results of interferon-y release assay and tuberculin skin test in routine screening for latent tuberculosis in patients with inflammatory bowel diseases. Inflamm Bowel Dis 2011; 17: 84-90. http://dx.doi.org/10.1002/ibd.21427

[16] Lamberti M, Uccello R, Monaco MGL, Muoio M, Feola D, Sannolo N, et al. Tuberculin skin test and Quantiferon test agreement and influencing factors in tuberculosis screening of healthcare workers: a systematic review and metaanalysis. J Occup Med Toxicol 2015; 10: 2. http://dx.doi.org/10.1186/s12995-015-0044-y

[17] Johnson JL, Geldenhuys H, Thiel BA, Toefy A, Suliman S, Pienaar $\mathrm{B}$, et al. Effect of isoniazid therapy on QuantiFeronTB Gold In-Tube responses in adults with positive tuberculin skin test results in a high TB incidence area. Chest 2014; 145: 612-7.

http://dx.doi.org/10.1378/chest.13-1232

[18] Guglielmetti L, Mazzaferri F, Cordioli M, Conti M, Lo Cascio G, Concia E, Cazzadori A, et al. Rebound in QuantiFERONTB Gold In-Tube response after active tuberculosis treatment. J Infect 2015; 70: 96-8.

\section{http://dx.doi.org/10.1016/i.jinf.2014.07.007}

[19] Chiappini E, Bonsignori F, Mangone G, Galli L, Mazzantini R, Sollai S, et al. Serial T-SPOT.TB and quantiFERON-TB-Gold In-Tube assays to monitor response to antitubercular treatment in Italian children with active or latent tuberculosis infection. Pediatr Infect Dis J 2012; 31: 974-7. http://dx.doi.org/10.1097/INF.0b013e31825d0d67

[20] Dyrhol-Riise AM, Gran G, Wentzel-Larsen T, Blomberg B, Haanshuus CG, Mørkve O. Diagnosis and follow-up of treatment of latent tuberculosis; the utility of the QuantiFERON-TB Gold In-tube assay in outpatients from a tuberculosis low-endemic country. BMC Infect Dis 2010; 10: 57.

\section{http://dx.doi.org/10.1186/1471-2334-10-57}

[21] Pollock NR, Kashino SS, Napolitano DR, Sloutsky A, Joshi S, Guillet $\mathrm{J}$, et al. Evaluation of the Effect of Treatment of Latent Tuberculosis Infection on QuantiFERON-TB Gold Assay Results. Infect Control Hosp Epidemiol 2009; 30: 392-5. http://dx.doi.org/10.1086/596606

[22] Ferebee SH. Controlled chemoprophylaxis trials in tuberculosis. A general review. Bibl Tuberc 1970; 26: 28-106.

[23] Linh NN, Marks GB, Crawford AB. Radiographic predictors of subsequent reactivation of tuberculosis. Int $\mathrm{J}$ Tuberc Lung Dis 2007; 11: 1136-42.

[24] Farhat M, Greenway C, Pai M, Menzies D. False-positive tuberculin skin tests: what is the absolute effect of BCG and non-tuberculous mycobacteria? Int J Tuberc Lung Dis 2006; 10: $1192-204$

[25] Tavast E, Tuuminen T, Pakkanen SH, Eriksson M, Kantele A, Järvinen A, et al. Immunosuppression adversely affects TST but not IGRAs in patients with psoriasis or inflammatory musculoskeletal diseases. Int J Rheumatol 2012; 2012: 381929

\section{http://dx.doi.org/10.1155/2012/381929}

[26] Encinales L, Zuñiga J, Granados-Montiel J, Yunis M, Granados J, Almeciga I, et al. Humoral immunity in tuberculin skin test anergy and its role in high-risk persons exposed to active tuberculosis. Mol Immunol 2010; 47: 1066-73. http://dx.doi.org/10.1016/j.molimm.2009.11.005

[27] Laffitte E, Janssens JP, Roux-Lombard P, Thielen AM, Barde $\mathrm{C}$, Marazza G, et al. Tuberculosis screening in patients with psoriasis before antitumour necrosis factor therapy: comparison of an interferon- $y$ release assay vs. tuberculin skin test. Br J Dermatol 2009; 161: 797-800. http://dx.doi.org/10.1111/j.1365-2133.2009.09331.x 
[28] Pratt A, Nicholl K, Kay L. Use of QuantiFERON TB Gold test as part of a screening programme in patients with RA under consideration for treatment with anti-TNF- $\alpha$ agents: the
Newcastle (UK) experience. Rheumatology 2007; 46: 1035-6.

http://dx.doi.org/10.1093/rheumatology/kem064

Received on 19-09-2015

Accepted on 24-11-2015

Published on 08-01-2016

http://dx.doi.org/10.6000/1929-6029.2016.05.01.7

(C) 2016 Dhaouadi et al.; Licensee Lifescience Global.

This is an open access article licensed under the terms of the Creative Commons Attribution Non-Commercial License (http://creativecommons.org/licenses/by-nc/3.0/) which permits unrestricted, non-commercial use, distribution and reproduction in any medium, provided the work is properly cited. 